\title{
A BPR METHODOLOGY FOR PRODUCT DEVELOPMENT PROCESSES
}

\author{
M. Bertoni ${ }^{1}$, U. Cugini ${ }^{1}$, D. Regazzoni ${ }^{2}$, C. Rizzi $^{2}$, M. Ugolotti ${ }^{1}$ \\ 'Dipartimento di Meccanica, Politecnico di Milano, Milano, Italy. \\ ${ }^{2}$ Dipartimento di Ingegneria Industriale, Università degli Studi di Bergamo, Dalmine $(B G)$, \\ Italy,Email: caterina.rizzi@unibg.it
}

\begin{abstract}
This paper presents methodology and guidelines about the use of process modeling languages to support BPR activities in relation with Product Development process. The methodology is based on the complementary adoption of different modeling techniques such as IDEF, UML and ARIS. Starting from the analysis of different models, we have selected and associated the most appropriate modeling suite to each phase of the re-engineering process. At this purpose, diagrams coming from IDEF, UML and ARIS families have been compared with respect to following issues: degree of formality, completeness, simplicity vs. detail, capability to describe "business goal" and support to costing operations.
\end{abstract}

Key words: Product development process, Business process re-engineering, Process modeling, IDEF, UML, ARIS.

\section{INTRODUCTION}

Those companies that consider product development a core competency, cannot ignore that during everyday work a big amount of information, in many different forms, is produced. This knowledge has to be captured, stored and correctly classified. At this purpose, PLM/PDM systems have been introduced in the last few years in order to manage the evolution of a product during its life cycle from the conception to the disposal [1]. When a PLM/PDM solution is designed and implemented, it has to be customized to best satisfy enterprise needs: main features are set and adapted in order to best fulfill company's process requirements. Thus, the design and implementation of a PLM solution requires an adequate analysis of the

Please use the following format when citing this chapter:

Bertoni, M., Cugini, U., Regazzoni, D., Rizzi C., Ugolotti, M., 2006, in International Federation for Information Processing (IFIP), Volume 207, Knowledge Enterprise: Intelligent Strategies In Product Design, Manufacturing, and Management, eds. K. Wang, Kovacs G., Wozny M., Fang M., (Boston: Springer), pp. 370-378. 
involved business processes. Often, such implementation is coupled with a Business Process Re-engineering (BPR) activity to better deploy technologies and/or methodologies [1][2]. A BPR project consists on a "fundamental rethinking and radical redesign of business processes to achieve dramatic improvement in critical, contemporary measures of performance such as cost, quality, service and speed" [3]. It can be seen as an iterative activity. After performing the initial AS-IS and TO-BE analysis of the company's process (step1), a new solution is physically implemented (step 2) and then, once the new system is working, it needs to be monitored and controlled (step 3). Detailed process description are used in order to guide and support all the activities performed along the "Re-engineering cycle". In such context, process modelling plays a very important role: models are used to understand how the current process is working (AS-IS models) and to give a preview about how process will behave once it will be re-engineered (TO-BE models). In order to increase BPR project's rate of success, modeling languages have to be carefully selected [4]. This choice is crucial, and not easy. The world of business modeling languages is characterized, in fact, by the lack of one leading business modelling technique. Models are heterogeneous and no official accepted standards exist in this area [5]. Many languages, like IDEF [6] and ARIS [7] are not equipped to support the description of software and IT systems. On the other hand, UML [8] is not specifically targeted for business-oriented models [9].

Starting from the concept of the Re-engineering cycle, main goal of this paper is to defined a methodology, named Integrated Methodology, and guidelines to be applied for the use of process modeling techniques in the context of BPR. We decided to focus on Product Development activity, basing on our previous experiences in this field [1][10]. The described methodology has been experimented within an industrial context in order to recognize and evaluate benefits and drawbacks. The study case we considered refers to the Product Development process of an Italian SME producing hydraulic systems [1]. Main goal was to implement a PLM/PDM system: decision taken in order to grant a better coordination and data exchange among company's department, facilitate testing and prototype activities and improve the product development lead-time. To have a complete overview of the entire design process, we considered all design process typologies described in the Total Quality Management procedures, where each type of design process is subdivided into four main phases: Concept generation, Preliminary design, Detailed design, Product engineering. 


\section{PROCESS VIEWS \& MODELLING TECHNIQUES}

Starting from the KAEMaRT methodology [10], we tried to define a new methodology for BPR project evaluating the possibility to use, in addition to IDEF models, different types of diagrams like those provided by ARIS and UML. The introduction of these modeling techniques suggested to enlarge AS-IS and TO-BE views, in order to represent with an higher level of completeness several aspects of enterprise processes. The modeling suites have been analyzed and compared realizing the AS-IS and TO-BE model of the considered Product Development process. Having the possibility to choose among different views allows the process analyst to work with the ad hoc instrument in relation with the context, the objective and re-engineering steps.

Five different views have been considered (Fig.1):

- PROCESS VISION. Models of this view are characterized by the smallest granularity in process description. From this perspective, an abstract process representation focused on macro-activities is given.

- WHAT. This view lets the analyst to describe WHAT the process does. It collects functional models, which represent information flows, considering all the other aspects of the process (time, data and products) that concur to function definitions.

- HOW. Diagrams related to this view permit to the user to describe HOW the process works. They provide a dynamic picture of system behavior providing a time sequenced description of the activity flows, in terms of parallelism and sequence relations.

- DATA/OBJECTS: This view concerns data, objects and all elements that contribute to data/objects definition. Models here are used to describe logical data structure, their state and their modification along the process flow.

- INTERACTIONS. These diagrams show patterns of interaction among people and objects that populate the system.

Figure 1 shows the diagrams we selected from the three modeling families for each process view. We could observe many similarities among models populating WHAT and HOW, both in terms of information collected and in terms of the graphical structure. IDEF0, Eriksson Penker BE, and ARIS Function View have been chosen in order to represent information's flows characterizing the WHAT row. At the same time, IDEF3 PF (Process Flow), UML Activity Diagrams, and ARIS EPC have been used to describe dynamic system's behavior. Data and objects were represented with IDEF 3 OSTN, with UML State and Class Diagrams and with ARIS ERM. UML Use Case Diagrams and ARIS Control View were elaborated in order to describe company's activities with minimum level of detail. Finally, thanks 
to UML Collaboration and Sequence Diagrams, we could represent message exchange between people and objects in the system.

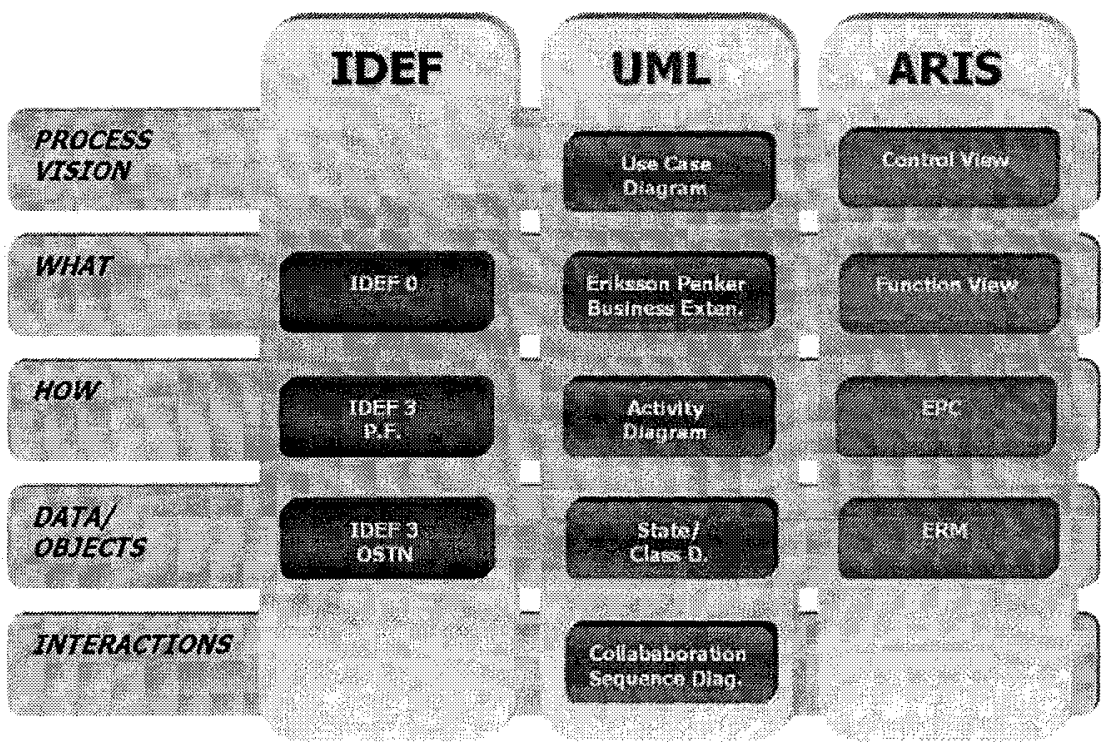

Figure 1. Process Modelling views and related modeling techniques.

\section{METHODOLGY FOR OPTIMAL CONTROL}

Both IDEF, UML and ARIS provide valuable tools in order to support BPR activity. Using diagrams from different methodologies we can horizontally enlarge our vision of the process, describing companies from five different points of view, and go deeper into the description of information, objects and activities flow. To define what kind of model best fulfill the requirements of each BPR step, we evaluated diagrams considering: Formality extent of the modeling language, Easiness of understanding vs. level of detail, Ability to describe business goals, Approach completeness, and Support to costing operations.

- Formality extent of the modeling language

A language is considered to be formal if its syntax and semantics can be precisely defined. When the semantics is formally defined, sentences in the language have a unique interpretation. Although these basic principles are common to most modeling techniques, the meaning of graphical symbols and the nature of their relations can be defined with a different level of 
precision. We can say that, in general, any business model should be easy and unambiguously readable by a person.

Selected IDEF techniques have a very precise syntax and semantics definitions [11]. Moreover, they are very simple and concise, easily readable also by non professionals of the modeling technique. On the other side, IDEF diagrams suffer from semantic limitations that narrow their use especially in the IT description field [12]. Syntax and semantics of UML diagrams are concisely defined [5]. However, due to its origin, UML is based on a semantic and syntax more software design oriented than business process oriented. ARIS suite contains an enormous quantity of graphical symbols, but its semantics is not very precisely defined [5]. For example, it's not so clear, what is the meaning of various connection types between orgunits in org-charts or connection types between functions and org-units in eEPC.

\section{- Easiness of understanding vs. level of detail}

When choosing a modeling technique, we have to keep well in mind who will read and use the models. For instance, during the AS-IS and TO-BE analysis models should be easily understandable by people with different competences and backgrounds; this facilitates communication and share of process knowledge. On the contrary, during the implementation step, the representations must be complete and well detailed to facilitate domain experts' work.

In general, IDEF notations are well suited to describe, with an high level of abstraction, the behavior of a very complex system thanks to the hierarchical decomposition of its diagrams [6]. IDEF models show to be inadequate when a detailed representation of enterprise processes, at the Implementation Description level is needed [12]. UML suite provides a very complete view of the company processes, particularly focalized on the IT aspects [13]. On the other side, newcomers can be disoriented in front of the formality and the intrinsic complexity of UML constructs. ARIS suite is composed by more or less 50 different diagrams [14], that can be used separately depending on the scope of the description. Therefore it can be used in many different ways, both by experts for a detailed analysis of the process or newcomers for a rough view of enterprise system.

\section{- Ability to describe business goals}

High level business goals as well as constraints and limitation influencing objectives achievement should be represented within the process diagrams to better address process analysis, especially during the evaluation of the status quo and the TO-BE definition.

IDEF modeling techniques do not contain specific constructs for the description of business system goals [15]. IDEF5 diagrams could be used at this purpose however they are poorly used [11]. UML suite comprises the 
specific Goal Model but, in practice, it's scarcely utilized [15]. Goals are anyway expressed inside UML Eriksson-Penker Business Extension providing a representation of business process completed with the description of "goal objects". High level goals description is best developed in ARIS. In fact, it proposes the Objective Diagram, which permit to describe goals, critical factors influencing the objectives and a list of functions supporting their achievement. However we cannot link goals list to the activity flows representation and Object Diagrams nevertheless give a clear description of the process situation and are not utilized in the context of enterprise BPR activities [11].

\section{- Approach completeness}

This aspect concerns the ability of modeling languages to provide an holistic view of the entire business process to be re-engineered.

IDEF modeling approach is largely function oriented [15]. It provides useful means to describe enterprise activity flows; in fact, IDEF notations have been merely designed with the aim to model company's system from a defined viewpoint, such as a "function viewpoint" or an "information viewpoint". This is both a strength and a weakness of this technique. Also considering that IDEF models are very useful to quickly represent processes behavior, on the other side, no effective means are provided in order to represent some key constituents of the process. UML modeling methodology provides about ten diagrams, which can be used to describe business process, choosing the level of granularity desired [15]. Depending on the scope we can decide to represent only aspects related to a precise perspective, selecting only the best suited set of models [16]. UML mainly provides suitable and widely-used modeling constructs for developing multiperspective descriptions of IT systems. ARIS house, as already said, comprises more than 50 diagrams [7][17], which enable users to investigate the process from many different points of view, choosing the detail extent best fitting the analysis scope. Anyway we could be disoriented in front of this big amount of tools.

\section{- Support provided to costing operations}

When dealing with product or process problem solving, another important issue is metrics: we must define and measure what we mean by improvement considering techniques and tools that permit to evaluate and report on productivity and innovation. For example, during the Conceptual step of the re-engineering cycle, analysts need to evaluate two or more alternatives in the field of cost effectiveness, in order to identify the solution with the highest input/output ratio.

IDEF language supports the $\mathrm{ABC}$ (Activity Based Costing) costing technique. $\mathrm{ABC}$ feature is typically defined only for IDEF0 diagrams but not for IDEF3, although this last is the most used business process models inside 
IDEF suite [15]. Due to its origin, UML suffers a lack of extensions dedicated to the cost effectiveness analysis. Parameters necessary to perform gap analysis cannot be correctly defined inside UML language. ARIS methodology is supported by a number of software components which support cost analysis, such as ARIS Process Cost Analyzer used to plan and analyse prices and allocation rates [7]. It support $\mathrm{ABC}$ costing technique and each function has a very rich list of cost attributes, which can be used to obtain total values either via static analysis or via simulation [11][18].

Figure 2 summarises previous considerations also in relation with process re engineering life-cycle.

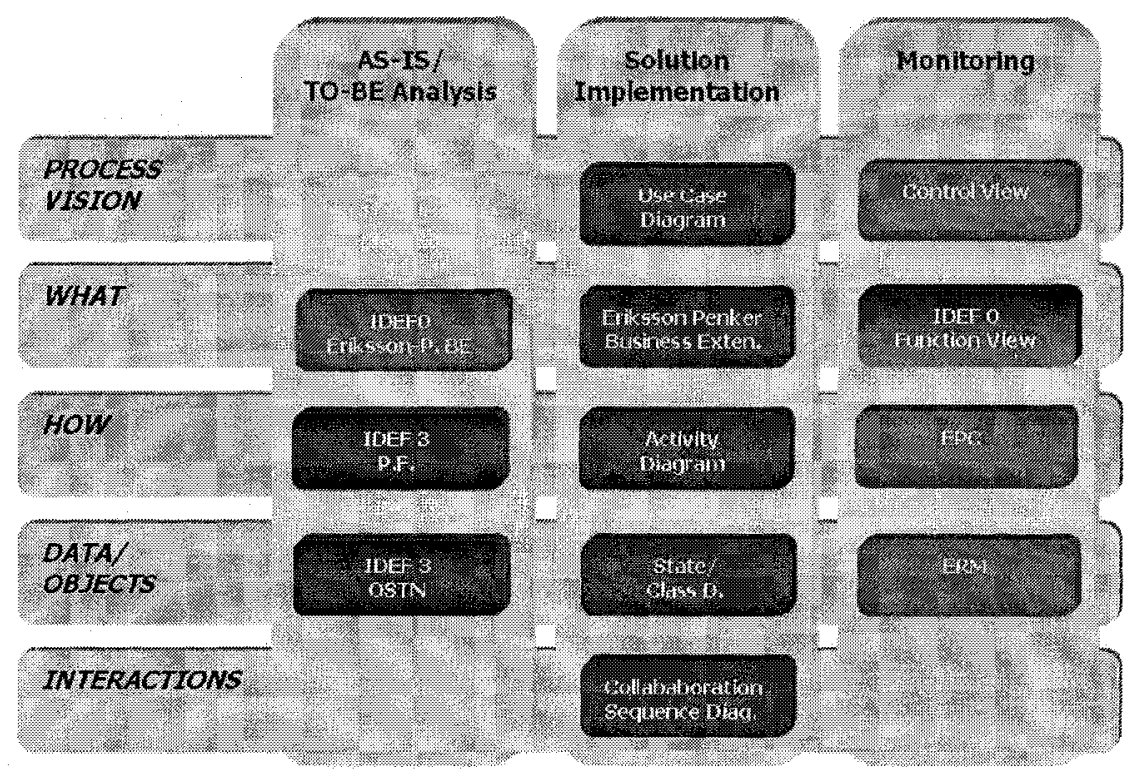

Figure 2. Proposed Integrated Methodology.

Conceptual analysis requires a clear and understandable representation of the enterprise process. Many people are involved coming from different company functions and with different backgrounds. Diagrams should be easily understandable, many process flows alternatives have be evaluated and representations should few detailed to allow an easy reading and a fast realization. IDEF techniques seem to be the perfect tools to provide a brief and concise description of the AS-IS and TO-BE models of the process to be re-engineered. However, in order to better share BPR objectives among different people and to motivate personnel, UML Eriksson-Penker BE can be used to represent the information flows during the Conceptual step. 
During the Implementation activity, process representations will be utilized by domain experts to implement the solution set in the previous step. Therefore models should describe in detail what the new system will do, how its different components will be combined and how they will work together. This BPR step is perfectly supported by UML diagrams.

In the Monitoring step, diagrams are mainly used by business people, not experts in the modeling techniques. The diagrams should be simple, to be easily understood also without a technical background, but, at the same time they should contain specific constructs to support gap analysis and costing operations. ARIS diagrams and IDEF0 seems to be the correct choice in order to support process description at this step.

\section{CONCLUSION}

Main scope of this work has been to develop a methodology and guidelines for the use of modeling languages during Product Development process re-engineering. In order to obtain a more realistic and reliable description of the process under analysis, we considered five different perspectives (views) and three different modeling suites. Choosing among different diagrams and depending on the scope of the description, one can focus the attention on data, objects, interactions, activity flows. Thanks to the Integrated Methodology it is possible to explicit the need of an holistic view of the company structure to abandon the narrow vision provided by the traditional approach, and to enlarge our understanding of the process, analyzing relationships and behaviors between processes inside the global enterprise.

Classifying IDEF, UML and ARIS diagrams inside the five different views, depending on the information they allow to represent, and comparing modeling techniques on the basis of the five aspects previously introduced, it was possible to detect most appropriate technique to be utilized in the three steps of the Re-engineering cycle.

Finally, we tried to realize a mapping among the information contained in models populating the same row of the table (Fig. 2). We evaluated the possibility to export information from a language to another, in order automate or semi-automate diagrams building up, with the scope to make feasible the adoption of the Integrated Methodology. It is concretely possible, making use of "neutral format", like .CSV files, opportunely translated through simple application written in $\mathrm{C}++$ code. Moreover, some special application has been recently developed in order to support, for instance, the unidirectional conversion from ARIS models to UML diagrams [19]. 


\section{REFERENCES}

1. Cugini, U., Ramelli, A., Rizzi, C., Ugolotti, M., (2004), PLM Paradigm in a SME: Total Quality Management and Process Modeling Techniques, in Proceeding $14^{\text {th }}$ International CIRP Design Seminar 2004 - Design in the Global Village (CD-ROM), Cairo (Egitto) 16-18 May 2004.

2. Scarbrough, H., (1997), Business Process Re-design: The knowledge dimension, Warwick Business School, University of Warwick, UK.

3. Hammer, M. and Champy, J., (1993), Reengineering the Corporation: A Manifesto for Business Revolution, Nicholas Brealey Publishing, London.

4. Childe, S., Maull R., Mills B., (1997), UK Experiences in Business Process Re engineering, University of Plymouth, UK.

5. Kalnis, A., Business Modelling. Languages and Tools, Institute of Mathematics and Computer Science, University of Latvia.

6. http://www.idef.com

7. ARIS toolset, 2000, www.a-cci.it

8. Bennett, B., Skelton, J., Lunn, K., (2001), Introduzione a UML, Ed. McGraw-Hill, UK.

9. Rittgen, P., Business Process in UML, Information System Group II, Technical University Darmstadt.

10. Rizzi, C., Ruozi, D., Gherri, N., (2004), A methodology to acquire and formalise process knowldege for technology innovation: an industrial application, Knowledge Intensive Design Technology, Eds J.C. Borg, P.J. Farrugia, K.P. Camilleri, 2004, Kluwer Academic Publishers, pp. 65-80.

11. Kalnins, A., Kalnina, D., Askolds Kalis, (1999), Comparison of Tools and Languages for Business Process Reengineering, Institute of Mathematics and Computer Science, University of Latvia, Latvia.

12. Cheol-Han, K., Weston, R.H. , Hodgson, A., Kyung-Huy L., (2001), The complementary use of IDEF and UML modelling approaches, Ed. Elsevier, UK.

13. Eriksson, H.E., Penker, M., (2000), Business modeling with UML, Ed . John Wiley and sons, INC, USA.

14. Scheer, A.W., Abolhassan, F., Jost, W., Kirchmer, M., (2002), Business process excellence, Aris in practice, Ed. Springer, GER.

15. Business modeling: UML vs. IDEF, Griffith University School of computing and information technology, (C) Ovidiu S. Noran.

16. Loos, P., Allweyer, T., (1998), Process orientation and Object orientation - An approach for integrating UML and Event Driven Process Chain $(E P C)$, Publication of the institute für Wirtscheftsinformatik, University of Saarland, Saarbriicken.

17. ARIS Methods Manual Version 4.1, AG Sheer, ED. IDS, July 1999.

18. Lewis, R.J., (1993), Activity Based Costing for Marketing and Manufacturing, Ed. Quorum Books.

19. ArcStyler MDA, Business Transformation Tutorial, ww.omg.org/mda. 